\title{
Electrolytes between dielectric charged surfaces: Simulations and theory
}

Cite as: J. Chem. Phys. 142, 194104 (2015); https://doi.org/10.1063/1.4921221

Submitted: 02 March 2015 . Accepted: 05 May 2015. Published Online: 18 May 2015

Alexandre P. dos Santos, and Yan Levin
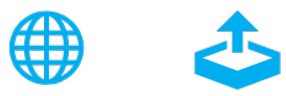

\section{ARTICLES YOU MAY BE INTERESTED IN}

Simulations of Coulomb systems with slab geometry using an efficient 3D Ewald summation method

The Journal of Chemical Physics 144, 144103 (2016); https://doi.org/10.1063/1.4945560

Ionic structure in liquids confined by dielectric interfaces

The Journal of Chemical Physics 143, 194508 (2015); https://doi.org/10.1063/1.4935704

Simulations of Coulomb systems confined by polarizable surfaces using periodic Green functions

The Journal of Chemical Physics 147, 184105 (2017); https://doi.org/10.1063/1.4997420

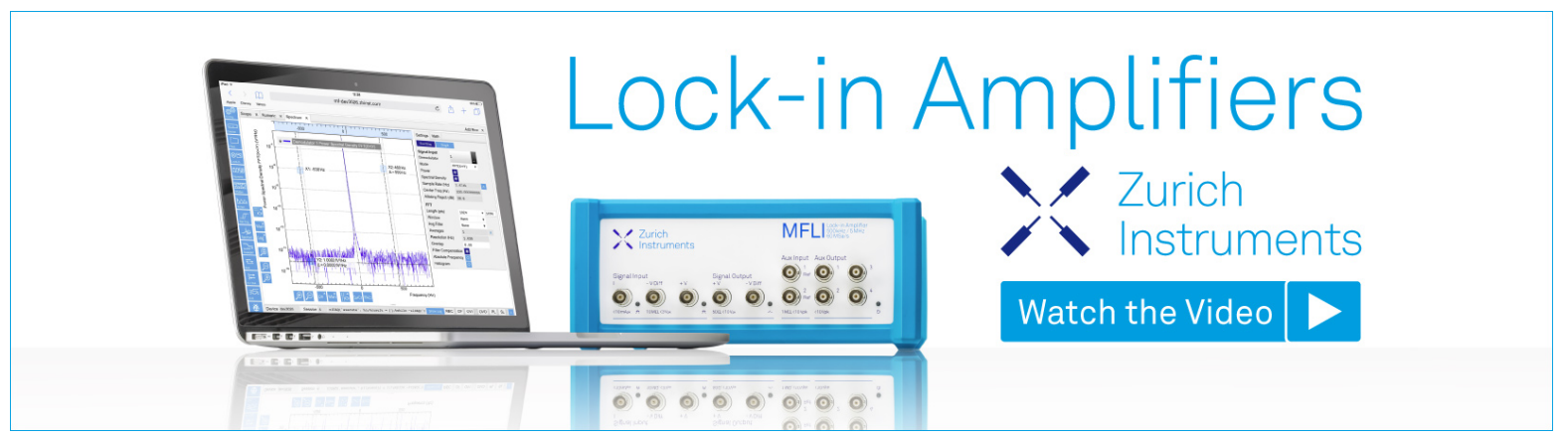




\title{
Electrolytes between dielectric charged surfaces: Simulations and theory
}

\author{
Alexandre P. dos Santos a) and Yan Levin ${ }^{\text {b) }}$ \\ Instituto de Física, Universidade Federal do Rio Grande do Sul, Caixa Postal 15051, \\ CEP 91501-970, Porto Alegre, RS, Brazil
}

(Received 2 March 2015; accepted 5 May 2015; published online 18 May 2015)

\begin{abstract}
We present a simulation method to study electrolyte solutions in a dielectric slab geometry using a modified 3D Ewald summation. The method is fast and easy to implement, allowing us to rapidly resum an infinite series of image charges. In the weak coupling limit, we also develop a mean-field theory which allows us to predict the ionic distribution between the dielectric charged plates. The agreement between both approaches, theoretical and simulational, is very good, validating both methods. Examples of ionic density profiles in the strong electrostatic coupling limit are also presented. Finally, we explore the confinement of charge asymmetric electrolytes between neutral surfaces. () 2015 AIP Publishing LLC. [http://dx.doi.org/10.1063/1.4921221]
\end{abstract}

\section{INTRODUCTION}

Complex charged liquids present a variety of interesting phenomena such as like-charge attraction ${ }^{1,2}$ and charge inversion. ${ }^{3-5}$ These phenomena arise as a result of electrostatic correlations of counterions in the double layer. ${ }^{6}$ To explore the ionic correlations between the overlapping double layers, one must have accurate and reliable methods to study electrolytes in a slab geometry. Similar difficulties are encountered when one wants to understand the thermodynamics of ionic liquid based supercapacitors. ${ }^{7}$

Electrolytes in a slab-like geometry have been extensively studied in the past. ${ }^{8-12}$ However, the dielectric contrast between the solvent and the confining surfaces is usually not taken into account. This dielectric mismatch leads to polarization of interfaces, which dramatically increases the mathematical complexity of the problem. For a single interface, the dielectric heterogeneity and the resulting induced surface charge can lead to a repulsion of electrolyte from the charged dielectric surface. ${ }^{13,14}$ Similar behavior has been observed near charged colloidal particles. ${ }^{15-21}$

Most of the theoretical and simulational works involving confined electrolytes neglect the dielectric contrast which results in an infinite series of image charges. While this significantly simplifies the calculations, it also fails to account for some of the fundamental physics of the overlapping double layers. Recently, Wang and Wang ${ }^{22}$ presented a meanfield theoretical discussion of confined electrolytes between charged and neutral plates. In the same year, Zwanikken and de la $\mathrm{Cruz}^{23}$ developed a liquid state theory which predicted that neutral confining polarizable interfaces can attract each other inside an electrolyte solution. Similar result can be found in other works. ${ }^{24-26}$ Samaj and Trizac ${ }^{27}$ and Jho et al. ${ }^{28}$ developed theories to study the distribution of confined counterions between charged plates, in a salt free system in a strong-coupling limit. Some studies focused on the specific case in which the dielectric constant of the surrounding medium is much

\footnotetext{
a) Electronic mail: alexandre.pereira@ufrgs.br

b)Electronic mail: levin@if.ufrgs.br
}

lower than of water. ${ }^{29-31}$ Jho et al. ${ }^{32}$ developed a simulation method for confined counterions based on the electrostatic layer correction (ELC) method. ${ }^{33}$ Also, Tyagi et al. ${ }^{34,35}$ constructed the ICMMM2D method which is the generalization of the MMM2D algorithm, ${ }^{36}$ previously developed to study homogeneous dielectric slab systems. Although these methods account for the surface polarization, they require a calculation of a sum of terms for the electrostatic potential that make simulations quite slow. Similar difficulties are encountered with other simulation approaches. ${ }^{26}$ Boundary element methods (BEM) consider the minimization of functionals and can be applied to systems with general geometries. Some BEM consider local polarization charge densities as dynamic variables, ${ }^{37,38}$ and others attempt to explicitly calculate the bound charge.$^{39,40}$ Even though BEM are expensive computationally, they have been extensively used to study general soft matter problems. ${ }^{41-43}$

In this paper, we present a simulation method based on 3D Ewald summation with a modified correction for the slab geometry. ${ }^{44}$ The method is simple to implement. It does not require summations of a slowly convergent infinite series of images during the simulation and is comparable in time with a regular 3D Ewald method. The paper is organized as follows. In Sec. II, we show how to construct the electrostatic energy of the system. In Sec. III, we study confined electrolytes between polarizable charged surfaces. In Sec. IV, we present a meanfield theory for 1:1 electrolytes and compare it with simulations. In Sec. V, we present the general results, and in Sec. VI, the conclusions.

\section{ELECTROSTATIC ENERGY}

To perform simulations, we use a rectangular simulation box of sides $L_{x}, L_{y}$, and $L_{z}$. The box contains $N$ ions of charges $q_{j}=\alpha_{j} Q$, where $\alpha_{j}$ is the valence of the ion and $Q$ is the proton charge, confined in the region $-L_{x} / 2<x<L_{x} / 2,-L_{y} / 2$ $<y<L_{y} / 2$, and $-L / 2<z<L / 2$. We set $L_{y}=L_{x}$ and $L_{z}$ $=5 L$. The uniform dielectric constants are $\epsilon_{w}$, inside the slab containing electrolyte, and $\epsilon_{o}$, outside. The dielectric contrast results in an infinite set of "images of images," which must be 


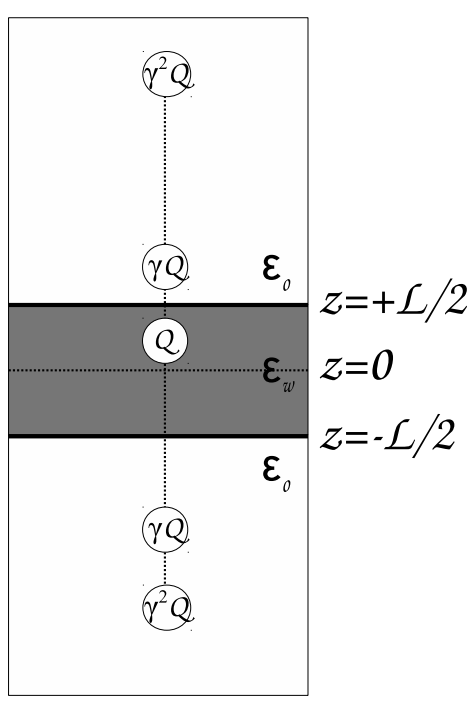

FIG. 1. Representation of a charge between the dielectric surfaces. Only the first and second order images are shown, $N_{i}=2$.

resummed to obtain the total electrostatic energy. We define $N_{i}$ as a number of images of an ion at each interface. To calculate the exact electrostatic energy, $N_{i}$ should be infinite. This, however, is not practical in a simulation. Instead, we explore the convergence of simulations as the number of images $N_{i}$ is increased. For example, if $N_{i}=2$, we consider one image charge at each dielectric interface and the image of image, producing 4 image charges for each ion, see Fig. 1. The electrostatic potential at the position $\boldsymbol{r}$ (in the region with $\epsilon_{w}$ ), created by all ions (excluding ion $i$ ), their image charges (including the image of ion $i$ ), and the periodic replicas is

$$
\begin{aligned}
\phi_{i}(\boldsymbol{r})= & \sum_{\boldsymbol{n}}^{\infty} \sum_{j=1}^{N} \int \frac{\rho_{j}(\boldsymbol{s})}{\epsilon_{w}|\boldsymbol{r}-\boldsymbol{s}|} d^{3} \boldsymbol{s} \\
& +\sum_{m=1}^{N_{i}} \sum_{n}^{\infty} \sum_{j=1}^{N}\left[\int \frac{\rho_{j m}^{+}(\boldsymbol{s})}{\epsilon_{w}|\boldsymbol{r}-\boldsymbol{s}|} d^{3} \boldsymbol{s}+\int \frac{\rho_{j m}^{-}(\boldsymbol{s})}{\epsilon_{w}|\boldsymbol{r}-\boldsymbol{s}|} d^{3} \boldsymbol{s}\right],
\end{aligned}
$$

where $\rho_{j}(\boldsymbol{s})=q_{j} \delta\left(\boldsymbol{s}-\boldsymbol{r}_{j}-\boldsymbol{r}_{e p}\right) \quad$ and $\quad \rho_{j m}^{ \pm}(\boldsymbol{s})=\gamma^{m} q_{j} \delta(\boldsymbol{s}$ $\left.-\boldsymbol{r}_{j m}^{ \pm}-\boldsymbol{r}_{e p}\right)$ are the charge densities of ions and their replicas and of dielectric images and their replicas.

The replication vector is defined as $\boldsymbol{r}_{e p}=L_{x} n_{x} \hat{\boldsymbol{x}}$ $+L_{y} n_{y} \hat{\boldsymbol{y}}+L_{z} n_{z} \hat{z}$ and $\boldsymbol{r}_{j m}^{ \pm}=x_{j} \hat{\boldsymbol{x}}+y_{j} \hat{\boldsymbol{y}}+\left[(-1)^{m} z_{j} \pm m L\right] \hat{\boldsymbol{z}}$. The vectors $\boldsymbol{n}=\left(n_{x}, n_{y}, n_{z}\right)$, where $n_{x}, n_{y}$, and $n_{z}$ are positive and negative integers, represent the infinite replicas of the main cell. The constant $\gamma$ is defined as $\gamma=\left(\epsilon_{w}-\epsilon_{o}\right) /\left(\epsilon_{w}+\epsilon_{o}\right)$ and the prime on the summation signifies that $j \neq i$, when $\boldsymbol{n}=(0,0,0)$. The total electrostatic energy of the system is

$$
U=\frac{1}{2} \sum_{i=1}^{N} q_{i} \phi_{i}\left(\boldsymbol{r}_{i}\right) .
$$

The energy above is very difficult to calculate because of a slow conditional convergence of the series in Eq. (1). To speed up the convergence, we use the Ewald method in which the ionic charge is partially screened by placing a Gaussiandistributed charge of opposite sign on top of each ion. ${ }^{45} \mathrm{We}$ then add and subtract an opposite Gaussian charge at the posi- tion of each ion and its images, $\rho_{j}(s)$ and $\rho_{j m}^{ \pm}(s)$, respectively. The potential, Eq. (1), then becomes

$$
\phi_{i}(\boldsymbol{r})=\phi_{i}^{S}(\boldsymbol{r})+\phi^{L}(\boldsymbol{r})-\phi_{i}^{\text {self }}(\boldsymbol{r}),
$$

where

$$
\begin{aligned}
\phi_{i}^{S}(\boldsymbol{r})= & \sum_{n}^{\infty} \sum_{j=1}^{N} \int \frac{\rho_{j}(\boldsymbol{s})-\rho_{j}^{G}(\boldsymbol{s})}{\epsilon_{w}|\boldsymbol{r}-\boldsymbol{s}|} d^{3} \boldsymbol{s} \\
& +\sum_{m=1}^{N_{i}} \sum_{\boldsymbol{n}}^{\infty} \sum_{j=1}^{N}\left[\int \frac{\rho_{j m}^{+}(\boldsymbol{s})-\rho_{j m}^{G+}(\boldsymbol{s})}{\epsilon_{w}|\boldsymbol{r}-\boldsymbol{s}|} d^{3} \boldsymbol{s}\right. \\
& \left.+\int \frac{\rho_{j m}^{-}(\boldsymbol{s})-\rho_{j m}^{G-}(\boldsymbol{s})}{\epsilon_{w}|\boldsymbol{r}-\boldsymbol{s}|} d^{3} \boldsymbol{s}\right] \\
\phi^{L}(\boldsymbol{r})= & \sum_{\boldsymbol{n}}^{\infty} \sum_{j=1}^{N} \int \frac{\rho_{j}^{G}(\boldsymbol{s})}{\epsilon_{w}|\boldsymbol{r}-\boldsymbol{s}|} d^{3} \boldsymbol{s} \\
& +\sum_{m=1}^{N_{i}} \sum_{\boldsymbol{n}}^{\infty} \sum_{j=1}^{N}\left[\int \frac{\rho_{j m}^{G+}(\boldsymbol{s})}{\epsilon_{w}|\boldsymbol{r}-\boldsymbol{s}|} d^{3} \boldsymbol{s}\right. \\
& \left.+\int \frac{\rho_{j m}^{G-}(\boldsymbol{s})}{\epsilon_{w}|\boldsymbol{r}-\boldsymbol{s}|} d^{3} \boldsymbol{s}\right]
\end{aligned}
$$

and

$$
\phi_{i}^{\text {self }}(\boldsymbol{r})=\int \frac{\rho_{i}^{G}(\boldsymbol{s})}{\epsilon_{w}|\boldsymbol{r}-\boldsymbol{s}|} d^{3} \boldsymbol{s}
$$

where

$$
\begin{gathered}
\rho_{j}^{G}(\boldsymbol{s})=q_{j}\left(\kappa_{e}^{3} / \sqrt{\pi^{3}}\right) \exp \left(-\kappa_{e}^{2}\left|\boldsymbol{s}-\boldsymbol{r}_{j}-\boldsymbol{r}_{e p}\right|^{2}\right), \\
\rho_{j m}^{G \pm}(\boldsymbol{s})=\gamma^{m} q_{j}\left(\kappa_{e}^{3} / \sqrt{\pi^{3}}\right) \exp \left(-\kappa_{e}^{2}\left|\boldsymbol{s}-\boldsymbol{r}_{j m}^{ \pm}-\boldsymbol{r}_{e p}\right|^{2}\right),
\end{gathered}
$$

and $\kappa_{e}$ is a damping parameter. Note that we have subtracted the self-potential, Eq. (6), from Eq. (3), in order to remove the prime over the summation in the long-range $(L)$ part of the potential, Eq. (5). The electrostatic potential produced by the Gaussian charges can be calculated using the Poisson equation, yielding

$$
\begin{aligned}
\phi^{L}(\boldsymbol{r})= & \sum_{\boldsymbol{n}}^{\infty} \sum_{j=1}^{N} q_{j} \frac{\operatorname{erf}\left(\kappa_{e}\left|\boldsymbol{r}-\boldsymbol{r}_{j}-\boldsymbol{r}_{e p}\right|\right)}{\epsilon_{w}\left|\boldsymbol{r}-\boldsymbol{r}_{j}-\boldsymbol{r}_{e p}\right|} \\
& +\sum_{m=1}^{N_{i}} \sum_{\boldsymbol{n}}^{\infty} \sum_{j=1}^{N} \gamma^{m} q_{j}\left[\frac{\operatorname{erf}\left(\kappa_{e}\left|\boldsymbol{r}-\boldsymbol{r}_{j m}^{+}-\boldsymbol{r}_{e p}\right|\right)}{\epsilon_{w}\left|\boldsymbol{r}-\boldsymbol{r}_{j m}^{+}-\boldsymbol{r}_{e p}\right|}\right. \\
& \left.+\frac{\operatorname{erf}\left(\kappa_{e}\left|\boldsymbol{r}-\boldsymbol{r}_{j m}^{-}-\boldsymbol{r}_{e p}\right|\right)}{\epsilon_{w}\left|\boldsymbol{r}-\boldsymbol{r}_{j m}^{-}-\boldsymbol{r}_{e p}\right|}\right]
\end{aligned}
$$

where $\operatorname{erf}(x)$ is the error function. The short-range part of the potential $(S)$, Eq. (4), can then be obtained in terms of the complementary error function, $\operatorname{erfc}(x)=1-\operatorname{erf}(x)$,

$$
\begin{aligned}
\phi_{i}^{S}(\boldsymbol{r})= & \sum_{\boldsymbol{n}}^{\infty} \sum_{j=1}^{N} q_{j} \frac{\operatorname{erfc}\left(\kappa_{e}\left|\boldsymbol{r}-\boldsymbol{r}_{j}-\boldsymbol{r}_{e p}\right|\right)}{\epsilon_{w}\left|\boldsymbol{r}-\boldsymbol{r}_{j}-\boldsymbol{r}_{e p}\right|} \\
& +\sum_{m=1}^{N_{i}} \sum_{\boldsymbol{n}}^{\infty} \sum_{j=1}^{N} \gamma^{m} q_{j}\left[\frac{\operatorname{erfc}\left(\kappa_{e}\left|\boldsymbol{r}-\boldsymbol{r}_{j m}^{+}-\boldsymbol{r}_{e p}\right|\right)}{\epsilon_{w}\left|\boldsymbol{r}-\boldsymbol{r}_{j m}^{+}-\boldsymbol{r}_{e p}\right|}\right. \\
& \left.+\frac{\operatorname{erfc}\left(\kappa_{e}\left|\boldsymbol{r}-\boldsymbol{r}_{j m}^{-}-\boldsymbol{r}_{e p}\right|\right)}{\epsilon_{w}\left|\boldsymbol{r}-\boldsymbol{r}_{j m}^{-}-\boldsymbol{r}_{e p}\right|}\right] .
\end{aligned}
$$


This potential decays exponentially fast, with the decay length controlled by the damping parameter which we set to $\kappa_{e}$ $=4 / R_{c}$, where $R_{c}=L_{x}$ is the distance cutoff. It is then necessary to consider only the term $\boldsymbol{n}=(0,0,0)$, with the usual periodic boundary condition. Furthermore, for sufficiently large values of $\kappa$, it is sufficient to include only a few imagesof-images. The damping parameter, however, cannot be too high since its value controls the number of $\boldsymbol{k}$-vectors that will have to be used to calculate the long-range potential. For systems studied in this paper, we find that $N_{i}=2$, in the short range potential, is sufficient. Depending on the separation between the plates, more images may be necessary. Prior to accumulation of data, we, therefore, check for convergence by varying the value of $N_{i}$. The short-range potential then becomes

$$
\begin{aligned}
\phi_{i}^{S}(\boldsymbol{r})= & \sum_{j=1}^{N} q_{j}^{\prime} \frac{\operatorname{erfc}\left(\kappa_{e}\left|\boldsymbol{r}-\boldsymbol{r}_{j}\right|\right)}{\epsilon_{w}\left|\boldsymbol{r}-\boldsymbol{r}_{j}\right|}+\sum_{m=1}^{2} \sum_{j=1}^{N} \gamma^{m} q_{j} \\
& \times\left[\frac{\operatorname{erfc}\left(\kappa_{e}\left|\boldsymbol{r}-\boldsymbol{r}_{j m}^{+}\right|\right)}{\epsilon_{w}\left|\boldsymbol{r}-\boldsymbol{r}_{j m}^{+}\right|}+\frac{\operatorname{erfc}\left(\kappa_{e}\left|\boldsymbol{r}-\boldsymbol{r}_{j m}^{-}\right|\right)}{\epsilon_{w}\left|\boldsymbol{r}-\boldsymbol{r}_{j m}^{-}\right|}\right] .
\end{aligned}
$$

The self-potential, Eq. (6), reduces to

$$
\phi_{i}^{\text {self }}(\boldsymbol{r})=q_{i} \frac{\operatorname{erf}\left(\kappa_{e}\left|\boldsymbol{r}-\boldsymbol{r}_{i}\right|\right)}{\epsilon_{w}\left|\boldsymbol{r}-\boldsymbol{r}_{i}\right|} .
$$

We next calculate the long-range part of the potential, Eq. (9). This is most easily obtained using the Fourier representation, $\hat{\phi}^{L}(\boldsymbol{k})=\int d^{3} \boldsymbol{r} \exp (-i \boldsymbol{k} \cdot \boldsymbol{r}) \phi^{L}(\boldsymbol{r})$, since in the reciprocal space, all sums, once again, converge very rapidly. The Fourier transform $\hat{\rho}^{T}(\boldsymbol{k})=\int d^{3} \boldsymbol{r} \exp (-i \boldsymbol{k} \cdot \boldsymbol{r}) \rho^{T}(\boldsymbol{r})$, of the Gaussian charge density,

$$
\begin{aligned}
\rho^{T}(\boldsymbol{r})= & \sum_{\boldsymbol{n}}^{\infty} \sum_{j=1}^{N} q_{j} \frac{\kappa_{e}^{3}}{\sqrt{\pi^{3}}} \exp \left(-\kappa_{e}^{2}\left|\boldsymbol{r}-\boldsymbol{r}_{j}-\boldsymbol{r}_{e p}\right|^{2}\right) \\
& +\sum_{m=1}^{N_{i}} \sum_{\boldsymbol{n}}^{\infty} \sum_{j=1}^{N} \gamma^{m} q_{j} \frac{\kappa_{e}^{3}}{\sqrt{\pi^{3}}}\left[\exp \left(-\kappa_{e}^{2}\left|\boldsymbol{r}-\boldsymbol{r}_{j m}^{+}-\boldsymbol{r}_{e p}\right|^{2}\right)\right. \\
& \left.+\exp \left(-\kappa_{e}^{2}\left|\boldsymbol{r}-\boldsymbol{r}_{j m}^{-}-\boldsymbol{r}_{e p}\right|^{2}\right)\right],
\end{aligned}
$$

is

$$
\hat{\rho}^{T}(\boldsymbol{k})=\frac{(2 \pi)^{3}}{V} \exp \left(-\frac{|\boldsymbol{k}|^{2}}{4 \kappa_{e}^{2}}\right)\left[\sum_{j=1}^{N} q_{j} \exp \left(-i \boldsymbol{k} \cdot \boldsymbol{r}_{j}\right)+\sum_{m=1}^{N_{i}} \sum_{j=1}^{N} \gamma^{m} q_{j}\left[\exp \left(-i \boldsymbol{k} \cdot \boldsymbol{r}_{j m}^{+}\right)+\exp \left(-i \boldsymbol{k} \cdot \boldsymbol{r}_{j m}^{-}\right)\right],\right.
$$

where $\boldsymbol{k}=\left(2 \pi n_{x} / L_{x y}, 2 \pi n_{y} / L_{x y}, 2 \pi n_{z} / L_{z}\right)$ and $V=L_{x} L_{y} L_{z}$. Using the Poisson equation, $|\boldsymbol{k}|^{2} \hat{\phi}^{L}(\boldsymbol{k})=\left(4 \pi / \epsilon_{w}\right) \hat{\rho}^{T}(\boldsymbol{k})$, we can evaluate the Fourier transform of the potential,

$$
\hat{\phi}^{L}(\boldsymbol{k})=\frac{8 \pi^{4}}{\epsilon_{w} V|\boldsymbol{k}|^{2}} \exp \left(-\frac{|\boldsymbol{k}|^{2}}{4 \kappa_{e}^{2}}\right)\left[\sum_{j=1}^{N} q_{j} \exp \left(-i \boldsymbol{k} \cdot \boldsymbol{r}_{j}\right)+\sum_{m=1}^{N_{i}} \sum_{j=1}^{N} \gamma^{m} q_{j}\left[\exp \left(-i \boldsymbol{k} \cdot \boldsymbol{r}_{j m}^{+}\right)+\exp \left(-i \boldsymbol{k} \cdot \boldsymbol{r}_{j m}^{-}\right)\right]\right] \text {. }
$$

The corresponding real-space electrostatic potential is calculated using the inverse Fourier transform, $\phi^{L}(\boldsymbol{r})=\frac{1}{(2 \pi)^{3}}$ $\sum_{\boldsymbol{k}} \hat{\phi}^{L}(\boldsymbol{k}) \exp (i \boldsymbol{k} \cdot \boldsymbol{r})$,

$$
\begin{aligned}
\phi^{L}(\boldsymbol{r})= & \sum_{\boldsymbol{k}} \frac{4 \pi}{\epsilon_{w} V|\boldsymbol{k}|^{2}} \exp \left(-\frac{|\boldsymbol{k}|^{2}}{4 \kappa_{e}^{2}}\right) \exp (i \boldsymbol{k} \cdot \boldsymbol{r}) \\
& \times\left[\sum_{j=1}^{N} q_{j} \exp \left(-i \boldsymbol{k} \cdot \boldsymbol{r}_{j}\right)+\sum_{m=1}^{N_{i}} \sum_{j=1}^{N} \gamma^{m} q_{j}\right. \\
& \left.\times\left[\exp \left(-i \boldsymbol{k} \cdot \boldsymbol{r}_{j m}^{+}\right)+\exp \left(-i \boldsymbol{k} \cdot \boldsymbol{r}_{j m}^{-}\right)\right]\right] .
\end{aligned}
$$

The long-range contribution to the total electrostatic energy is then given by $U_{L}=(1 / 2) \sum_{i=1}^{N} q_{i} \phi^{L}\left(\boldsymbol{r}_{i}\right)$, where $\phi^{L}(\boldsymbol{r})$ is obtained from Eq. (16). It is convenient to rewrite this in terms of functions

$$
\begin{gathered}
A(\boldsymbol{k})=\sum_{i=1}^{N} q_{i} \cos \left(\boldsymbol{k} \cdot \boldsymbol{r}_{i}\right), \\
B(\boldsymbol{k})=-\sum_{i=1}^{N} q_{i} \sin \left(\boldsymbol{k} \cdot \boldsymbol{r}_{i}\right),
\end{gathered}
$$

$$
C(\boldsymbol{k})=\sum_{m=1}^{N_{i}} \sum_{i=1}^{N} \gamma^{m} q_{i}\left[\cos \left(\boldsymbol{k} \cdot \boldsymbol{r}_{i m}^{+}\right)+\cos \left(\boldsymbol{k} \cdot \boldsymbol{r}_{i m}^{-}\right)\right]
$$

and

$$
D(\boldsymbol{k})=-\sum_{m=1}^{N_{i}} \sum_{i=1}^{N} \gamma^{m} q_{i}\left[\sin \left(\boldsymbol{k} \cdot \boldsymbol{r}_{i m}^{+}\right)+\sin \left(\boldsymbol{k} \cdot \boldsymbol{r}_{i m}^{-}\right)\right]
$$

The electrostatic energy then becomes

$$
\begin{aligned}
U_{L}= & \sum_{\boldsymbol{k}} \frac{2 \pi}{\epsilon_{w} V|\boldsymbol{k}|^{2}} \exp \left(-\frac{|\boldsymbol{k}|^{2}}{4 \kappa_{e}^{2}}\right) \\
& \times\left[A(\boldsymbol{k})^{2}+B(\boldsymbol{k})^{2}+A(\boldsymbol{k}) C(\boldsymbol{k})+B(\boldsymbol{k}) D(\boldsymbol{k})\right] .
\end{aligned}
$$

The terms in Eqs. (19) and (20) are multiplied by the $\gamma^{m}$ parameter, leading to a converging sum for realistic $\gamma<1$ parameter values. However, we do not know a priori a minimum number of images necessary to obtain an accurate result for the longrange potential. For example, we find that for $\gamma \approx 0.9$, we need $N_{i}=50$ to obtain a good convergence. However, such a large number of images make simulations extremely slow. We note, 
however, that Eqs. (19) and (20) can be rewritten as

$$
\begin{aligned}
C(\boldsymbol{k})= & \sum_{i=1}^{N} q_{i}\left[c_{1}(\boldsymbol{k}) \cos \left(k_{x} x_{i}+k_{y} y_{i}\right) \cos \left(k_{z} z_{i}\right)\right. \\
& \left.+c_{2}(\boldsymbol{k}) \sin \left(k_{x} x_{i}+k_{y} y_{i}\right) \sin \left(k_{z} z_{i}\right)\right]
\end{aligned}
$$

and

$$
\begin{aligned}
D(\boldsymbol{k})= & -\sum_{i=1}^{N} q_{i}\left[d_{1}(\boldsymbol{k}) \sin \left(k_{x} x_{i}+k_{y} y_{i}-k_{z} z_{i}\right)\right. \\
& \left.+d_{2}(\boldsymbol{k}) \sin \left(k_{x} x_{i}+k_{y} y_{i}+k_{z} z_{i}\right)\right]
\end{aligned}
$$

where

$$
\begin{gathered}
c_{1}(\boldsymbol{k})=2 \sum_{m=1}^{N_{i}} \gamma^{m} \cos \left(m k_{z} L\right), \\
c_{2}(\boldsymbol{k})=2 \sum_{m=1}^{N_{i}}(-1)^{m+1} \gamma^{m} \cos \left(m k_{z} L\right), \\
d_{1}(\boldsymbol{k})=2 \sum_{m_{o}=1}^{N_{i}} \gamma^{m_{o}} \cos \left(m_{o} k_{z} L\right), \\
d_{2}(\boldsymbol{k})=2 \sum_{m_{e}=2}^{N_{i}} \gamma^{m_{e}} \cos \left(m_{e} k_{z} L\right),
\end{gathered}
$$

and $m$ are integers: $m_{o}$ are odd and $m_{e}$ are even. The parameters $c_{1}(\boldsymbol{k}), c_{2}(\boldsymbol{k}), d_{1}(\boldsymbol{k})$, and $d_{2}(\boldsymbol{k})$ can be obtained once (up to any desired accuracy) at the beginning of the simulation since they do not depend on the ionic positions. The functions, $A(\boldsymbol{k})$, $B(\boldsymbol{k}), C(\boldsymbol{k})$, and $D(\boldsymbol{k})$, can now be easily updated for each new configuration in a Monte Carlo (MC) simulation.

The electrostatic energy resulting from the short-range part of the potential is $U_{S}=(1 / 2) \sum_{i=1}^{N} q_{i} \phi_{i}^{S}\left(\boldsymbol{r}_{i}\right)$, where $\phi_{i}^{S}(\boldsymbol{r})$ is given by the Eq. (11), and the self-energy contribution is $U_{\text {self }}=(1 / 2) \sum_{i=1}^{N} q_{i} \phi_{i}^{\text {self }}\left(\boldsymbol{r}_{i}\right)$. In the limit $x \rightarrow 0$, the $\operatorname{erf}(x)$ function vanishes as $(2 / \sqrt{\pi}) x$ and the self-energy contribution reduces to $U_{\text {self }}=\left(\kappa_{e} / \epsilon_{w} \sqrt{\pi}\right) \sum_{i=1}^{N} q_{i}^{2}$. The total electrostatic interaction energy of the ions is given by the expressions above, plus the correction needed to account for the slab geometry. ${ }^{44}$

Yeh and Berkowitz ${ }^{44}$ found that a regular 3D Ewald summation method with an energy correction which accounts for the anisotropic summation of a conditionally convergent series in a slab-like geometry can reproduce the same results as the 2D Ewald method, with a significant gain in performance. For more details on the Ewald summation method, applied to different geometries, an interested reader can consult Refs. 46-48. Following Yeh and Berkowitz ${ }^{44}$ and Smith $^{49}$ and taking into account the dielectric discontinuity and the induced image charges, we find the correction for the slab geometry to be

$$
\begin{aligned}
U_{c o r}= & -\frac{\pi}{\epsilon_{w} V} \sum_{i=1}^{N} q_{i}\left[\sum_{j=1}^{N} q_{j}\left(z_{i}-z_{j}\right)^{2}\right. \\
& \left.+\sum_{m=1}^{N_{i}} \sum_{j=1}^{N} \gamma^{m} q_{j}\left[\left(z_{i}-z_{j m}^{-}\right)^{2}+\left(z_{i}-z_{j m}^{+}\right)^{2}\right]\right],
\end{aligned}
$$

where $z_{j m}^{ \pm}=(-1)^{m} z_{j} \pm m L$. Using the electroneutrality, this expression can be rewritten as

$$
U_{c o r}=\frac{2 \pi}{\epsilon_{w} V} M_{z}^{2}\left[1+2 \sum_{m=1}^{N_{i}}(-\gamma)^{m}\right],
$$

where $M_{z}=\sum_{i=1}^{N} q_{i} z_{i}$ is the magnetization in the $\hat{z}$ direction. Again, the constant between the brackets can be evaluated once at the beginning of the simulation, so that $M_{z}$ can be easily updated in the simulation process. The total energy of the system is then

$$
U=U_{S}+U_{L}+U_{\text {cor }}+U_{\text {self }} .
$$

\section{MONTE CARLO SIMULATIONS}

We now study an electrolyte solution confined between two negatively charged dielectric surfaces. The two charged plates contain 256 point charges each, uniformly distributed on the surface on a square lattice. The magnitude of point charges is adjusted to obtain the desired surface charge density. The surfaces are located at $z=-L / 2$ and $z=+L / 2$. Counterions are modeled as hard spheres with the charge $q$ located at the center. Besides the counterions, salt ions can also be present in the system, all with the same ionic radius, $2 \AA$. The solvent is modeled as a uniform dielectric medium. The Bjerrum length $\lambda_{B}=\beta Q^{2} / \epsilon_{w}$ of the system is $7.2 \AA$, corresponding to water at room temperature. The $\mathrm{MC}$ simulations are performed using the Metropolis algorithm. The method developed in Sec. II is used to obtain the electrostatic energy. Care must be taken in the calculation of the electrostatic energy of wall particles. For these particles, the self-image electrostatic interaction diverges, leading to an infinite constant which must be renormalized. We use $1 \times 10^{6}$ attempted particle moves to equilibrate and 100 moves per particle to create a new state. After 40000 states, we calculate the average ionic density profiles.

\section{MODIFIED POISSON-BOLTZMANN (MPB) EQUATION}

To test the simulation method developed above, we compare the results with a mPB equation. The $\mathrm{mPB}$ equation is constructed to account approximately for the ion-image and charge-charge correlations near an interface and is expected to work well in the weak coupling limit. It was tested against MC simulation for a single dielectric interface with 1:1 electrolyte and was found to be very accurate. Therefore, we expect that for a slab geometry, a suitably modified $\mathrm{mPB}$ equation will also remain very accurate, allowing us to test the new simulation method.

The electrostatic potential between two negatively charged dielectric surfaces satisfies the exact Poisson equation

$$
\nabla^{2} \phi(z)=-\frac{4 \pi}{\epsilon_{w}}\left[Q \rho_{+}(z)-Q \rho_{-}(z)\right],
$$

where $\phi(z)$ is the mean electrostatic potential at a distance $z$ from the first plate (for simplicity it is placed at $z=0$ ), and $\rho_{+}(z)$ and $\rho_{-}(z)$ are the concentrations of cations and anions derived from salt and surface dissociations. In equilibrium, all 
the ions will be distributed in accordance with the Boltzmann distribution, $\exp \left(-\beta \omega_{s}\right)$, where $\omega_{s}$ is the potential of mean force of ion of type $s$. We will approximate $\omega_{s}$ by the meanelectrostatic potential plus the correlation contribution, $W(z)$. Suppose that there are $N_{+}$cations, and $N_{-}$anions per square Angstrom, their distributions are then given by

$$
\begin{aligned}
& \rho_{+}(z)=N_{+} \frac{e^{-\beta Q \phi(z)-\beta W(z)}}{\int_{r_{c}}^{L-r_{c}} d z e^{-\beta Q \phi(z)-\beta W(z)}}, \\
& \rho_{-}(z)=N_{-} \frac{e^{\beta Q \phi(z)-\beta W(z)}}{\int_{r_{c}}^{L-r_{c}} d z e^{\beta Q \phi(z)-\beta W(z)}} .
\end{aligned}
$$

If the expression for $W(z)$ is known, we can solve the mPB equation numerically to obtain the ionic density profiles.

The correlational and charge-image contribution $W(z)$ can be calculated approximately as follows. Consider $N_{+}$ions (per $\AA^{2}$ ), with charge $Q$, and $N_{-}$ions (per $\AA^{2}$ ), with charge $-Q$, all with hydration radii $r_{c}$, confined between two neutral dielectric walls with separation $L$, see Fig. 2. Due to the hardcore repulsion of ions from the surfaces, they are restricted to the regions $z>r_{c}$ and $z<L-r_{c}$. To keep the charge neutrality of this region, we introduce a uniform neutralizing background charge density $-Q N_{c} /\left(L-2 r_{c}\right)$, where $N_{c}=N_{+}-N_{-}$. In the exterior regions $z<0$ and $z>L$, the dielectric constant is $\epsilon_{o}$, while in the interior region, it is $\epsilon_{w}$. The function $W(z)$ then corresponds, approximately, to the energy penalty that an ion located at a distance $z$ from one of the surfaces feels due to asymmetry of its ionic atmosphere and due to its hard core repulsion from the wall. To obtain this potential, we calculate the Green's function for a system of differential equations: Laplace equation,

$$
\nabla^{2} \phi(\mathbf{s}, z)=0
$$

in the region with no electrolyte and a linearized PoissonBoltzmann (LPB) equation,

$$
\nabla^{2} \phi(\mathbf{s}, z)=\kappa^{2} \phi(\mathbf{s}, z)
$$

in the region accessible to ions, ${ }^{50}$ where $\phi(\mathbf{s}, z)$ is the potential at position $(\mathbf{s}, z)$ in cylindrical coordinates and

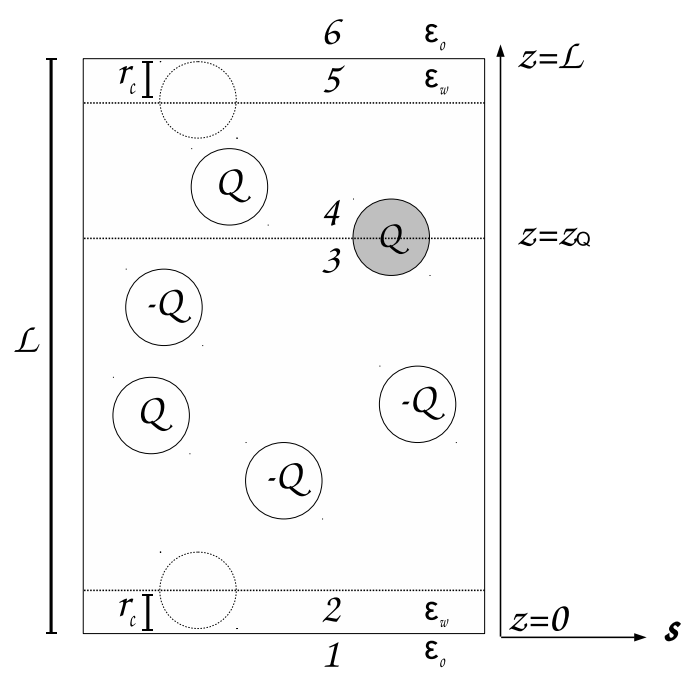

FIG. 2. Representation of an electrolyte in the region $r_{c}<z<L-r_{c}$ used to calculate $W(z)$. $\kappa=\sqrt{4 \pi \lambda_{B}\left(N_{+}+N_{-}\right) / L}$ is the inverse Debye length. LPB equation is used to account for the electrostatic correlations between the ions. ${ }^{6}$ For 1:1 electrolyte, linearization of the Poisson-Boltzmann equation is justified since the ionic interactions are weak. Because of the azimuthal symmetry of the problem, it is convenient to work with the Fourier transform of the potential, $\hat{\phi}(\mathbf{k}, z)$, defined in terms of ${ }^{50}$

$$
\phi(\mathbf{s}, z)=\frac{1}{2 \pi} \int_{0}^{\infty} d k k J_{0}(k s) \hat{\phi}(\mathbf{k}, z) .
$$

For dielectric interfaces between hydrocarbons and water, $\epsilon_{o} / \epsilon_{w} \ll 1$, so that to leading order we can set $\epsilon_{o}=0$. Taking into account the continuity of the electrostatic potential and of the normal component of the displacement field at $z=0$, $z=r_{c}, z=z_{Q}, z=L-r_{c}$, and $z=L$, the Fourier transform of the electrostatic potential in the region 3, see Fig. 2, can be calculated to be

$$
\hat{\phi}_{3}(\mathbf{k}, z)=A_{3} e^{p z}+B_{3} e^{-p z},
$$

where $\mathbf{k}$ is the wave vector and $p=\sqrt{\kappa^{2}+k^{2}}$. The constants are given by

$$
\begin{aligned}
& A_{3}=\frac{\pi Q f_{1}}{\epsilon_{w} f_{3}}\left(e^{p z_{Q}}\left[(p+k) e^{2 r_{c} p-r_{c} k}+(p-k) e^{2 r_{c} p+r_{c} k}\right]\right. \\
& \left.+e^{-p z_{Q}}\left[(p+k) e^{2 L p+r_{c} k}+(p-k) e^{2 L p-r_{c} k}\right]\right), \\
& B_{3}=A_{3} e^{2 r_{c} p} \frac{f_{2}}{f_{1}}, \\
& f_{1}=p \cosh \left(k r_{c}\right)+k \sinh \left(k r_{c}\right) \text {, } \\
& f_{2}=p \cosh \left(k r_{c}\right)-k \sinh \left(k r_{c}\right) \text {, } \\
& f_{3}=p\left(e^{2 L p} f_{1}^{2}-e^{4 r_{c} p} f_{2}^{2}\right) \text {. }
\end{aligned}
$$

Considering $s \rightarrow 0$ and $z \rightarrow z_{Q}$, we find (now omitting subscript 3)

$$
\begin{aligned}
\phi\left(z_{Q}\right)= & \frac{Q}{2 \epsilon_{w}} \int_{0}^{\infty} d k k\left(\frac { f _ { 1 } } { f _ { 3 } } e ^ { 2 p z _ { Q } } \left[(p+k) e^{2 r_{c} p-r_{c} k}\right.\right. \\
& \left.+(p-k) e^{2 r_{c} p+r_{c} k}\right]+\frac{f_{2}}{f_{3}} e^{-2 p z} Q^{+2 r_{c} p} \\
& \left.\times\left[(p+k) e^{2 L p+r_{c} k}+(p-k) e^{2 L p-r_{c} k}\right]\right)+ \text { func, }
\end{aligned}
$$

where func does not depend on the ion-plate distance $z_{Q}$ and can be ignored. The work necessary to insert an ion at position $z=z_{Q}$ from the interface can be calculated using the Güntelberg $^{51}$ charging process

$$
\begin{aligned}
W\left(z_{Q}\right)= & \frac{Q^{2}}{4 \epsilon_{w}} \int_{0}^{\infty} d k k\left(\frac { f _ { 1 } } { f _ { 3 } } e ^ { 2 p z _ { Q } } \left[(p+k) e^{2 r_{c} p-r_{c} k}\right.\right. \\
& \left.+(p-k) e^{2 r_{c} p+r_{c} k}\right]+\frac{f_{2}}{f_{3}} e^{-2 p z} Q^{+2 r_{c} p} \\
& \left.\times\left[(p+k) e^{2 L p+r_{c} k}+(p-k) e^{2 L p-r_{c} k}\right]\right) .
\end{aligned}
$$

The interaction potential $W(z)$ can now be used in the $\mathrm{mPB}$ equation, Eq. (29), to account for the charge-image interaction and the polarization of the ionic atmosphere.

\section{RESULTS}

We first show the comparison between the ionic concentrations obtained using $\mathrm{mPB}$ Eq. (29) and the results of MC 

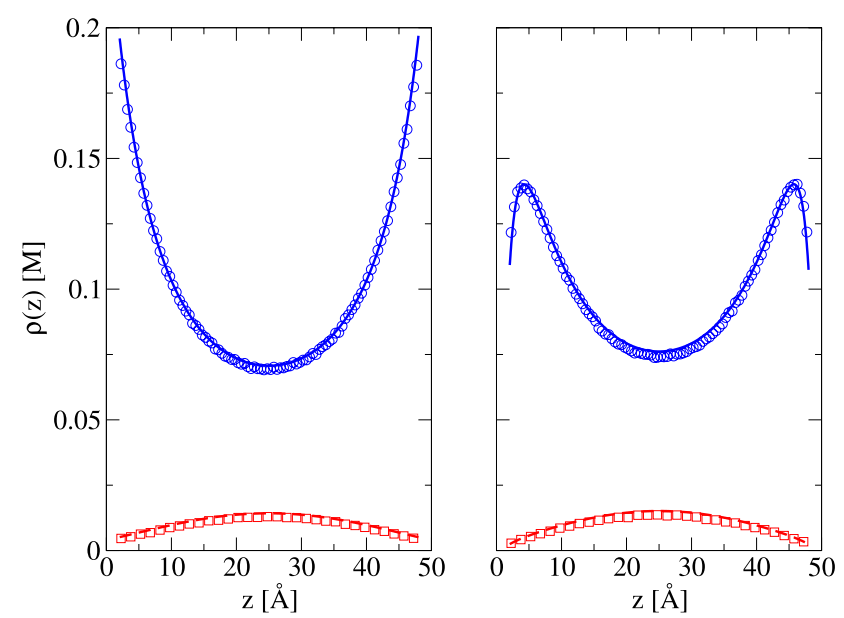

FIG. 3. Ionic density profiles for the cases: $\epsilon_{o}=\epsilon_{w}=80$, left, and $\epsilon_{o}=2$ and $\epsilon_{w}=80$, right. The plate charge density is $-0.02 \mathrm{C} / \mathrm{m}^{2}$, the distance between plates is $L=50 \AA$, and the monovalent salt concentration is $10 \mathrm{mM}$. Symbols represent the simulation data and lines represent the solution of the $\mathrm{mPB}$ equation, Eq. (29). Solid lines and circles represent cations, while dashed lines and squares represent the anions.

simulations. In Fig. 3, we show the ionic density profiles between two interfaces for the cases: (1) where $\epsilon_{o}=2$ and $\epsilon_{w}$ $=80$, for which $W(z)$ is approximately given by Eq. (37), and (2) where $\epsilon_{o}=\epsilon_{w}=80$, for which $W(z)$ is zero. The agreement between theory and simulations is very good, validating both methods.

We next explore the effects of the dielectric heterogeneity for strongly correlated systems. The valency of the counterions is modified to $\alpha_{j}=3$ and $\alpha_{j}=5$. The other parameters are kept the same as before. If $\epsilon_{w}=\epsilon_{o}$, most of the multivalent ions adsorb to the charged wall forming a strongly correlated quasi-2D one component plasma, see Fig. 4. However, in the case of large dielectric contrast between solvent and the confining surface, the counterions experience a strong charge-image repulsion from the surface, see Fig. 4. This can significantly affect the interaction between charged dielectric bodies inside an electrolyte solution. ${ }^{22}$ Finally, in Fig. 5, we show the

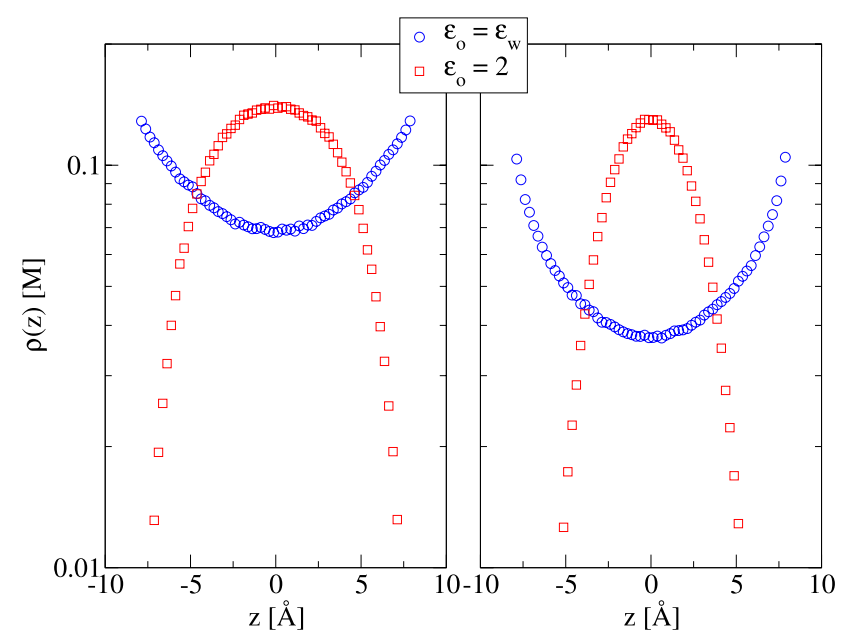

FIG. 4. Counterion density profile for the cases $\alpha_{j}=3$, left, and $\alpha_{j}=5$, right. The plate charge density is $-0.02 \mathrm{C} / \mathrm{m}^{2}$, and the distance between plates is $L=20 \AA$.
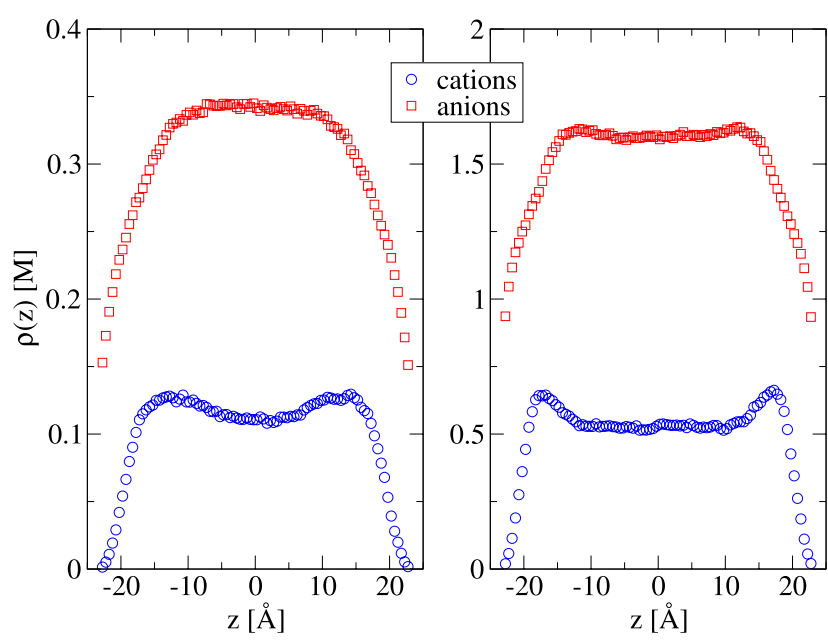

FIG. 5. Ionic density profiles for $\epsilon_{o}=2$ and 3:1 salt confined between neutral surfaces, for various salt concentrations. On the left, $100 \mathrm{mM}$ of salt, while on the right, $500 \mathrm{mM}$. The distance between plates is $L=50 \AA$. Circles represent trivalent cations and squares represent monovalent anions.

ionic distribution for 3:1 electrolyte between neutral dielectric surfaces.

\section{CONCLUSIONS}

In this paper, we developed a new simulation approach to study electrolytes and ionic liquids in a dielectric slab geometry. The method is easy to implement and is comparable in time consumption with the regular 3D Ewald summation method. In the weak coupling limit, we also presented a meanfield theory which allows us to predict the ionic distribution between the dielectric charged plates. The agreement between both approaches, theoretical and simulational, is very good, validating both methods. Examples of ionic density profiles for strongly correlated systems are also presented. Finally, the simulation method developed here can be used to explore the interactions between colloidal particles with strong dielectric contrast. This will be the subject of the future work.

\section{ACKNOWLEDGMENTS}

This work was partially supported by the CNPq, INCTFCx, and by the US-AFOSR under the Grant No. FA9550-121-0438.

${ }^{1}$ R. Kjellander and S. Marcelja, J. Phys. Chem. 90, 1230 (1986).

${ }^{2}$ P. Linse and V. Lobaskin, Phys. Rev. Lett. 83, 4208 (1999).

${ }^{3}$ A. Fernandez-Nieves, A. Fernandez-Barbero, F. J. de las Nieves, and B. Vincent, J. Chem. Phys. 123, 054905 (2005).

${ }^{4}$ S. Pianegonda, M. C. Barbosa, and Y. Levin, Europhys. Lett. 71, 831 (2005).

${ }^{5}$ A. P. dos Santos and Y. Levin, Phys. Rev. Lett. 106, 167801 (2011).

${ }^{6}$ Y. Levin, Rep. Prog. Phys. 65, 1577 (2002).

${ }^{7}$ Y. Shim, H. J. Kim, and Y. Jung, Faraday Discuss. 154, 249 (2012).

${ }^{8}$ I. Rouzina and V. Bloomfield, J. Chem. Phys. 100, 9977 (1996).

${ }^{9}$ B. I. Shklovskii, Phys. Rev. E 60, 5802 (1999).

${ }^{10}$ R. R. Netz, Eur. Phys. J. E 5, 557 (2001).

${ }^{11}$ A. G. Moreira and R. R. Netz, Eur. Phys. J. E 8, 33 (2002).

${ }^{12}$ L. Samaj and E. Trizac, Phys. Rev. Lett. 106, 078301 (2011).

${ }^{13}$ A. G. Moreira and R. R. Netz, Europhys. Lett. 52, 705 (2000).

${ }^{14}$ A. P. dos Santos and Y. Levin, Electrostatics of Soft and Disordered Matter (CRC Press, Boca Raton, 2014), Chap. 14, p. 181

${ }^{15}$ R. Messina, J. Chem. Phys. 117, 11062 (2002). 
${ }^{16}$ A. P. dos Santos, A. Bakhshandeh, and Y. Levin, J. Chem. Phys. 135, 044124 (2011).

${ }^{17}$ A. Bakhshandeh, A. P. dos Santos, and Y. Levin, Phys. Rev. Lett. 107, 107801 (2011).

${ }^{18}$ L. Lue and P. Linse, J. Chem. Phys. 135, 224508 (2011).

${ }^{19}$ A. Diehl, A. P. dos Santos, and Y. Levin, J. Phys.: Condens. Matter 24, 284115 (2012).

${ }^{20}$ V. Jadhao, F. J. Solis, and M. O. de la Cruz, J. Chem. Phys. 138, 054119 (2013).

${ }^{21}$ G. I. G. Garcia and M. O. de la Cruz, J. Phys. Chem. B 118, 8854 (2014).

${ }^{22}$ R. Wang and Z. G. Wang, J. Chem. Phys. 139, 124702 (2013).

${ }^{23}$ J. W. Zwanikken and M. O. de la Cruz, P. Natl. Acad. Sci. USA 110, 5301 (2013).

${ }^{24}$ G. M. Bell and S. Levine, J. Chem. Phys. 49, 4584 (1968).

${ }^{25}$ R. Kjellander and S. Marcelja, Chem. Phys. Lett. 142, 485 (1987).

${ }^{26}$ M. Kanduc, A. Naji, J. Forsman, and R. Podgornik, J. Chem. Phys. 137, 174704 (2012).

${ }^{27}$ L. Samaj and E. Trizac, Europhys. Lett. 100, 56005 (2012).

${ }^{28}$ Y. S. Jho, M. Kanduc, A. Naji, R. Podgornik, M. W. Kim, and P. A. Pincus, Phys. Rev. Lett. 101, 188101 (2008).

${ }^{29}$ J. Hautman, J. W. Halley, and Y. J. Rhee, J. Chem. Phys. 91, 467 (1989).

${ }^{30}$ J. W. Perram and M. A. Ratner, J. Chem. Phys. 104, 5174 (1996).

${ }^{31}$ S. H. L. Klapp, Mol. Simul. 32, 609 (2006).

${ }^{32}$ Y. S. Jho, G. Park, C. S. Chang, P. A. Pincus, and M. Kim, Phys. Rev. E 76, 011920 (2007).
${ }^{33}$ A. Arnold, J. Joannis, and C. Holm, J. Chem. Phys. 117, 2496 (2002).

${ }^{34}$ S. Tyagi, A. Arnold, and C. Holm, J. Chem. Phys. 127, 154723 (2007).

${ }^{35}$ S. Tyagi, A. Arnold, and C. Holm, J. Chem. Phys. 129, 204102 (2008).

${ }^{36}$ A. Arnold and C. Holm, Chem. Phys. Lett. 354, 324 (2002).

${ }^{37}$ M. Marchi, D. Borgis, N. Levy, and P. Ballone, J. Chem. Phys. 114, 4377 (2001).

${ }^{38}$ V. Jadhao, F. J. Solis, and M. O. de la Cruz, Phys. Rev. Lett. 109, 223905 (2012).

${ }^{39}$ D. Boda, D. Gillespie, W. Nonner, D. Henderson, and B. Eisenberg, Phys. Rev. E 69, 046702 (2004).

${ }^{40}$ K. Barros, D. Sinkovits, and E. Luijten, J. Chem. Phys. 140, 064903 (2014).

${ }^{41}$ T. Nagy, D. Henderson, and D. Boda, J. Phys. Chem. B 115, 11409 (2011).

${ }^{42}$ D. Boda, D. Henderson, and D. Gillespie, J. Chem. Phys. 139, 055103 (2013).

${ }^{43}$ C. Berti, S. Furini, D. Gillespie, D. Boda, R. S. Eisenberg, E. Sangiorgi, and C. Fiegna, J. Chem. Theory Comput. 10, 2911 (2014).

${ }^{44}$ I. C. Yeh and M. L. Berkowitz, J. Chem. Phys. 111, 3155 (1999).

${ }^{45}$ M. P. Allen and D. J. Tildesley, Computer Simulations of Liquids (Oxford University Press, Oxford, New York, 1987).

${ }^{46}$ E. Spohr, J. Chem. Phys. 107, 6342 (1997).

${ }^{47}$ E. R. Smith, J. Chem. Phys. 128, 174104 (2008).

${ }^{48}$ V. Ballenegger, J. Chem. Phys. 140, 161102 (2014).

${ }^{49}$ E. R. Smith, Proc. R. Soc. London A 375, 475 (1981).

${ }^{50}$ Y. Levin and J. E. Flores-Mena, Europhys. Lett. 56, 187 (2001).

${ }^{51}$ E. Z. Güntelberg, Z. Phys. Chem. 123, 199 (1926). 